\title{
Glutamate Dehydrogenase Measurement
}

National Cancer Institute

\section{Source}

National Cancer Institute. Glutamate Dehydrogenase Measurement. NCI Thesaurus.

Code C79448.

The determination of the amount of glutamate dehydrogenase present in a sample. 